\title{
AN HMM COMPENSATION APPROACH FOR DYNAMIC FEATURES USING UNSCENTED TRANSFORMATION AND ITS APPLICATION TO NOISY SPEECH RECOGNITION
}

\author{
$\mathrm{Yu} H u^{1}$ and Qiang $H u o^{2}$ \\ ${ }^{1}$ Department of Electronic Engineering \& Information Science, University of Science and Technology of China, Hefei, China \\ ${ }^{2}$ Microsoft Research Asia, Beijing, China \\ (E-mails: jadefox@ustc.edu qianghuo@microsoft.com)
}

\begin{abstract}
In our previous work, a new HMM compensation approach for static MFCC features was proposed by using a technique called Unscented Transformation (UT). Three implementations of the UT approach with different computational complexities were evaluated on Aurora 2 connected digits database, and significant performance improvements were achieved compared to log-normalapproximation-based PMC (Parallel Model Combination) and firstorder-approximation-based VTS (Vector Taylor Series) approaches. In this paper, we extend our UT-based formulation to compensating for HMM parameters corresponding to both static and dynamic features. New experimental results on Aurora2 task are reported to demonstrate the effectiveness of the proposed UT approach.
\end{abstract}

Index Terms: robust speech recognition, unscented transformation, model compensation, dynamic feature, hidden Markov model.

\section{INTRODUCTION}

Most of current automatic speech recognition (ASR) systems use MFCCs (Mel-Frequency Cepstral Coefficients) and their derivatives (e.g., delta, $\Delta$, and delta-delta, $\Delta^{2}$ ) as speech features, and a set of Gaussian mixture continuous density HMMs (CDHMMs) for modeling basic speech units (e.g., [9]). It is well known that the performance of such an ASR system trained with clean speech will degrade significantly when the testing speech is distorted by additive noises. How to achieve the noise robustness in the above scenario has been an important research topic in ASR field.

Among many approaches proposed previously to cope with the above robust ASR problem, two notable HMM compensation approaches are Parallel Model Combination (PMC) (e.g., [2]) and Vector Taylor Series (VTS) (e.g., [7, 8, 6, 1]) approaches, respectively. For both approaches, the following simplified assumptions are made: 1) The speech and noise signals are independent, and additive in the time domain; 2) The alignment between a speech frame and the corresponding Gaussian component of CDHMM used to train the speech models from the clean speech data is not altered by the addition of noise; 3) The Gaussian PDF (probability density function) remains appropriate for modeling the feature vectors of noisy speech aligned to the corresponding component. Having made the above assumptions, the problem of HMM compensation is simplified as a problem of how to calculate the mean vector and covariance matrix, $\left\{\mu_{\mathbf{y}}, \Sigma_{\mathbf{y}}\right\}$, for each Gaussian component of the noisy speech from the corresponding statistics $\left\{\mu_{\mathbf{x}}, \Sigma_{\mathbf{x}}\right\}$ for clean speech and $\left\{\mu_{\mathbf{n}}, \Sigma_{\mathbf{n}}\right\}$ for noise, respectively.

Even after the above simplification, the expressions for estimating the corrupted speech model parameters do not have closed- form solutions. Various approximations have previously been proposed to solve this problem. For PMC approach, numerical integration and data-driven PMC are two techniques that provide an accurate approximation but is computationally expensive, while the so-called log-normal and log-add approximations are less accurate but computationally more efficient [2]. For VTS approach [7, 8], a truncated (typically up to the first-order) Taylor series expansion is used to approximate the nonlinear distortion function that relates feature vectors of noisy speech to the ones of clean speech and noise. In [4], we proposed a new approach to addressing the above problem by using a technique called Unscented Transformation (UT) [5]. Three implementations of the UT approach with different computational complexities were evaluated on Aurora 2 connected digits database, and significant performance improvements were achieved compared to the log-normalapproximation-based PMC and the first-order-approximation-based VTS approaches. Although both the CDHMM mean and variance parameters for static MFCCs were compensated systematically by using the PMC-, VTS-, and UT-based approaches, only CDHMM mean parameters corresponding to dynamic features were compensated by using yet a more heuristic approach described in [1]. Encouraged by the promising results achieved in our previous study, we have extended our UT-based formulation to compensating for CDHMM parameters (both means and variances) corresponding to both static and dynamic features. It is the purpose of this paper that reports our study on this topic.

The rest of the paper is organized as follows. In Section 2, we present the formulation of our UT-based approach. In Section 3 , we report the experimental results, and finally we conclude the paper in Section 4.

\section{HMM COMPENSATION USING UNSCENTED TRANSFORMATION (UT)}

\subsection{Some Notations and Distortion Functions}

In this study, only additive noise is considered and the effect of channel distortion is ignored. Therefore, the distortion function between static features of clean and noisy speech in log-spectral domain can be expressed as:

$$
Y_{k}^{\log }=f\left(X_{k}^{\log }, N_{k}^{\log }\right)=\log \left(\exp \left(X_{k}^{\log }\right)+\exp \left(N_{k}^{\log }\right)\right)
$$

where $Y_{k}^{\log }, X_{k}^{\log }$ and $N_{k}^{\log }$ are the $k$ th component of random static feature vectors, $\mathbf{Y}$ for noisy speech, $\mathbf{X}$ for clean speech, and $\mathbf{N}$ for additive noise, respectively; and the superscript $\log$ indicates 
that the above feature vectors are extracted in the log-spectral domain. In the following, we will formulate our problem in the logspectral domain, therefore the superscript $\log$ will be omitted if no confusion will be caused according to the context of relevant discussions.

Although the delta and delta-delta features are extracted using linear regression method in most state-of-the-art ASR systems as described in e.g., [9], as an approximation, we derive the distortion function for delta features by taking a time derivative to the distortion function of static features in Eq. (1) as follows:

$$
\frac{\partial Y_{k}}{\partial t}=\frac{1}{1+\exp \left(U_{k}\right)} \frac{\partial X_{k}}{\partial t}+\frac{\exp \left(U_{k}\right)}{1+\exp \left(U_{k}\right)} \frac{\partial N_{k}}{\partial t},
$$

where $U_{k}$ is the $k$ th component of the random vector $\mathbf{U}=\mathbf{N}-\mathbf{X}$. Apparently, when $\mathbf{X}$ and $\mathbf{N}$ are two independent $K$-dimensional random vectors with normal PDFs with means and covariance matrices, $\left\{\mu_{\mathbf{x}}, \Sigma_{\mathbf{x}}\right\}$ and $\left\{\mu_{\mathbf{n}}, \Sigma_{\mathbf{n}}\right\}$, respectively, $\mathbf{U}$ has a normal PDF with mean $\mu_{\mathbf{u}}=\mu_{\mathbf{n}}-\mu_{\mathbf{x}}$ and covariance matrix $\Sigma_{\mathbf{u}}=\Sigma_{\mathbf{n}}+\Sigma_{\mathbf{x}}$. For the convenience of notation, the distortion function for deltafeatures can then be represented as follows:

$$
\Delta \mathbf{Y}=\frac{\mathbf{1}}{\mathbf{1}+\exp (\mathbf{U})} \bullet \Delta \mathbf{X}+\frac{\exp (\mathbf{U})}{\mathbf{1}+\exp (\mathbf{U})} \bullet \Delta \mathbf{N},
$$

where $\mathbf{1}$ is a $K$-dimensional constant vector with all elements being $1, \Delta \mathbf{X}$ and $\Delta \mathbf{N}$ are random delta-feature vectors for clean speech and additive noise respectively. In the above equation, the $\bullet$ operation denotes an element-wise product for two vectors or two matrices (will be used later). Similarly, the division of two vectors and the exponential function of a vector also take the corresponding element-wise operations accordingly.

The distortion function for delta-delta features $\Delta^{2} \mathbf{Y}$ can be derived in a similar way as follows:

$$
\begin{aligned}
\Delta^{2} \mathbf{Y}= & \frac{\mathbf{1}}{\mathbf{1}+\exp (\mathbf{U})} \bullet \Delta^{2} \mathbf{X}+\frac{\exp (\mathbf{U})}{\mathbf{1}+\exp (\mathbf{U})} \bullet \Delta^{2} \mathbf{N} \\
& +\frac{\exp (\mathbf{U})}{(\mathbf{1}+\exp (\mathbf{U}))^{2}} \bullet \Delta \mathbf{U} \bullet \Delta \mathbf{U},
\end{aligned}
$$

where $(\cdot)^{2}$ denotes an element-wise square of a vector, $\Delta^{2} \mathbf{X}$ and $\Delta^{2} \mathbf{N}$ are random delta-delta feature vectors for clean speech and additive noise respectively, and $\Delta \mathbf{U}=\Delta \mathbf{N}-\Delta \mathbf{X}$. Apparently, when $\Delta \mathbf{X}$ and $\Delta \mathbf{N}$ are two independent $K$-dimensional random vectors with normal PDFs with means and covariance matrices, $\left\{\mu_{\Delta \mathbf{x}}, \Sigma_{\Delta \mathbf{x}}\right\}$ and $\left\{\mu_{\Delta \mathbf{n}}, \Sigma_{\Delta \mathbf{n}}\right\}$, respectively, $\Delta \mathbf{U}$ has a normal PDF with mean $\mu_{\Delta \mathbf{u}}=\mu_{\Delta \mathbf{n}}-\mu_{\Delta \mathbf{x}}$ and covariance matrix $\Sigma_{\Delta \mathbf{u}}=$ $\Sigma_{\Delta \mathbf{n}}+\Sigma_{\Delta \mathbf{x}}$.

\subsection{UT Approach with Augmented Random Vectors}

In [4], a UT approach with augmented random vectors (referred to as Method 1 in [4]) was proposed to compensate for HMM means and variances corresponding to static features by using the distortion function in Eq. (1). In the following, we derive a similar UT approach to compensating for HMM means and variances corresponding to dynamic features.

For delta features, we can form an augmented $3 K$-dimensional random vector, $\mathbf{X}_{1}^{a}=\left[\Delta \mathbf{X}^{T}, \Delta \mathbf{N}^{T}, \mathbf{U}^{T}\right]^{T}$ with the following mean vector $\mu_{1}^{a}$ and covariance matrix $\Sigma_{1}^{a}$ :

$\mu_{1}^{a}=\left[\mu_{\Delta \mathbf{x}}{ }^{T}, \mu_{\Delta \mathbf{n}}{ }^{T}, \mu_{\mathbf{u}}{ }^{T}\right]^{T}, \Sigma_{1}^{a}=\left[\begin{array}{ccc}\Sigma_{\Delta \mathbf{x}} & \mathbf{0}_{K \times K} & \Sigma_{\Delta \mathbf{x u}} \\ \mathbf{0}_{K \times K} & \Sigma_{\Delta \mathbf{n}} & \Sigma_{\Delta \mathbf{n u}} \\ \Sigma_{\mathbf{u} \Delta \mathbf{x}} & \Sigma_{\mathbf{u} \Delta \mathbf{n}} & \Sigma_{\mathbf{u}}\end{array}\right]$, where the sub-matrix $\Sigma_{\mathbf{a b}}$ denotes a covariance matrix of two random vectors $\mathbf{A}$ and $\mathbf{B}$. For delta-delta features, we can form an augmented $4 K$-dimensional random vector $\mathbf{X}_{2}^{a}=\left[\Delta^{2} \mathbf{X}^{T}, \Delta^{2} \mathbf{N}^{T}, \mathbf{U}^{T}\right.$, $\left.\Delta \mathbf{U}^{T}\right]^{T}$ with the following mean vector $\mu_{2}^{a}$ and covariance matrix $\Sigma_{2}^{a}$ :

$$
\begin{aligned}
& \mu_{2}^{a}=\left[\mu_{\Delta^{2} \mathbf{x}}{ }^{T}, \mu_{\Delta^{2} \mathbf{n}^{T}}{ }^{T}, \mu_{\mathbf{u}}{ }^{T}, \mu_{\Delta \mathbf{u}}{ }^{T}\right]^{T}, \\
& \Sigma_{2}^{a}=\left[\begin{array}{llll}
\Sigma_{\Delta^{2} \mathbf{x}} & \mathbf{0}_{K \times K} & \Sigma_{\Delta^{2} \mathbf{x u}} & \Sigma_{\Delta^{2} \mathbf{x} \Delta \mathbf{u}} \\
\mathbf{0}_{K \times K} & \Sigma_{\Delta^{2} \mathbf{n}} & \Sigma_{\Delta^{2} \mathbf{n u}} & \Sigma_{\Delta^{2} \mathbf{n} \Delta \mathbf{u}} \\
\Sigma_{\mathbf{u} \Delta^{2} \mathbf{x}} & \Sigma_{\mathbf{u} \Delta^{2} \mathbf{n}} & \Sigma_{\mathbf{u}} & \Sigma_{\mathbf{u} \Delta \mathbf{u}} \\
\Sigma_{\Delta \mathbf{u} \Delta^{2} \mathbf{x}} & \Sigma_{\Delta \mathbf{u} \Delta^{2} \mathbf{n}} & \Sigma_{\Delta \mathbf{u u}} & \Sigma_{\Delta \mathbf{u}}
\end{array}\right] .
\end{aligned}
$$

Given $\left\{\mu_{1}^{a}, \Sigma_{1}^{a}\right\},\left\{\mu_{2}^{a}, \Sigma_{2}^{a}\right\}$, and the distortion functions in Eqs. (3) and (4), a standard UT procedure as described in [4] can be used to estimate $\left\{\mu_{\Delta \mathbf{y}}, \Sigma_{\Delta \mathbf{y}}\right\}$ and $\left\{\mu_{\Delta^{2} \mathbf{y}}, \Sigma_{\Delta^{2} \mathbf{y}}\right\}$ accordingly.

It is well-known that the computational cost of the UT approach is proportional to the number of sigma points, which depends on the dimension of the random vector concerned [5]. The above augmented UT approach is apparently not computationally efficient, therefore not used in our experiments. Instead, we develop a more efficient UT approach in the following subsection by making some simplified assumptions.

\subsection{A Simplified UT Approach to HMM Compensation for Dynamic Features}

Let's first define the following four new random vectors:

$$
\begin{aligned}
\mathbf{W}=\Delta \mathbf{U} \bullet \Delta \mathbf{U}, & \mathbf{O}=\frac{\mathbf{1}}{\mathbf{1}+\exp (\mathbf{U})}, \\
\mathbf{E}=\frac{\exp (\mathbf{U})}{\mathbf{1}+\exp (\mathbf{U})}, & \mathbf{S}=\frac{\exp (\mathbf{U})}{(\mathbf{1}+\exp (\mathbf{U}))^{2}} .
\end{aligned}
$$

If we assume that $\mathbf{X}, \Delta \mathbf{X}, \Delta^{2} \mathbf{X}, \mathbf{N}, \Delta \mathbf{N}, \Delta^{2} \mathbf{N}$ are uncorrelated, we can deduce that the following pairs of random vectors, $\{\mathbf{O}, \Delta \mathbf{X}\}$, $\{\mathbf{E}, \Delta \mathbf{N}\},\left\{\mathbf{O}, \Delta^{2} \mathbf{X}\right\},\left\{\mathbf{E}, \Delta^{2} \mathbf{N}\right\},\{\mathbf{S}, \mathbf{W}\}$ are also uncorrelated. We further assume that the PDFs of the above four random vectors can be approximated by four Gaussian PDFs with means and covariance matrices as $\left\{\mu_{\mathbf{w}}, \Sigma_{\mathbf{w}}\right\},\left\{\mu_{\mathbf{0}}, \Sigma_{\mathbf{o}}\right\},\left\{\mu_{\mathbf{e}}, \Sigma_{\mathbf{e}}\right\},\left\{\mu_{\mathbf{s}}, \Sigma_{\mathbf{s}}\right\}$, respectively.

Substituting the newly defined random vectors in Eq. (7) to Eq. (3), the distortion function for delta features can be written as

$$
\Delta \mathbf{Y}=\mathbf{O} \bullet \Delta \mathbf{X}+\mathbf{E} \bullet \Delta \mathbf{N} .
$$

It can then be derived that the mean vector and covariance matrix for delta features of noisy speech can be compensated as follows:

$$
\begin{aligned}
\mu_{\Delta \mathbf{y}}= & \mu_{\mathbf{0}} \bullet \mu_{\Delta \mathbf{x}}+\mu_{\mathrm{e}} \bullet \mu_{\Delta \mathbf{n}}, \\
\Sigma_{\Delta \mathbf{y}}= & \Sigma_{\mathbf{0} \bullet \Delta \mathbf{x}}+\Sigma_{\mathbf{e} \bullet \Delta \mathbf{n}}+\operatorname{Cov}(\mathbf{O} \bullet \Delta \mathbf{X}, \mathbf{E} \bullet \Delta \mathbf{N}) \\
& +\operatorname{Cov}(\mathbf{E} \bullet \Delta \mathbf{N}, \mathbf{O} \bullet \Delta \mathbf{X}),
\end{aligned}
$$

where

$$
\begin{aligned}
\mu_{\mathbf{e}} & =\mathbf{1}-\mu_{\mathbf{o}}, \\
\Sigma_{\mathbf{e}} & =\Sigma_{\mathbf{o}}, \\
\Sigma_{\mathbf{o} \bullet \Delta \mathbf{x}} & =\Sigma_{\mathbf{o}} \bullet \Sigma_{\Delta \mathbf{x}}+\mu_{\mathbf{o}} \mu_{\mathbf{o}}^{T} \bullet \Sigma_{\Delta \mathbf{x}}+\Sigma_{\mathbf{o}} \bullet \mu_{\Delta \mathbf{x}} \mu_{\Delta \mathbf{x}}^{T}, \\
\Sigma_{\mathbf{e} \bullet \Delta \mathbf{n}} & =\Sigma_{\mathbf{e}} \bullet \Sigma_{\Delta \mathbf{n}}+\mu_{\mathbf{e}} \mu_{\mathbf{e}}^{T} \bullet \Sigma_{\Delta \mathbf{n}}+\Sigma_{\mathbf{e}} \bullet \mu_{\Delta \mathbf{n}} \mu_{\Delta \mathbf{n}}^{T},
\end{aligned}
$$

and

$$
\begin{aligned}
\operatorname{Cov}(\mathbf{O} \bullet \Delta \mathbf{X}, \mathbf{E} \bullet \Delta \mathbf{N}) & =(\operatorname{Cov}(\mathbf{E} \bullet \Delta \mathbf{N}, \mathbf{O} \bullet \Delta \mathbf{X}))^{T} \\
& =-\Sigma_{\mathbf{o}} \bullet \mu_{\Delta \mathbf{X}} \mu_{\Delta \mathbf{N}}^{T} .
\end{aligned}
$$


Table 1. Summary of different methods in two sets of experiments.

\begin{tabular}{llll}
\hline & \multicolumn{3}{c}{ Methods } \\
\cline { 2 - 4 } Index & Static & Delta & Delta-delta \\
\hline$\# 1$ & baseline & baseline & baseline \\
$\# 2$ & Log-Normal PMC & Mean [1] & Mean [1] \\
$\# 3$ & First-Order VTS & Mean [1] & Mean [1] \\
$\# 4$ & Augmented UT & Mean [1] & Mean [1] \\
$\# 5$ & Hybrid UT\&VTS & Mean [1] & Mean [1] \\
$\# 6$ & Simplex UT & Mean [1] & Mean [1] \\
\hline$\# 7$ & Augmented UT & Mean [1] & \\
$\# 8$ & Augmented UT & Mean\&Var [1] & \\
$\# 9$ & Augmented UT & Simplified UT & \\
$\# 10$ & Augmented UT & Mean [1] & Mean [1] \\
$\# 11$ & Augmented UT & Mean\&Var [1] & Mean\&Var [1] \\
$\# 12$ & Augmented UT & Simplified UT & Simplified UT \\
$\# 13$ & Augmented UT & Simplified UT & Mean [1] \\
\hline
\end{tabular}

In the above equations, $\mu_{\mathbf{0}}$ and $\Sigma_{\mathbf{0}}$ can be estimated by using UT approach. We use a computationally efficient simplex UT approach which requires the number of sigma points proportional to the number of static MFCC features in the cepstral domain [4].

Similarly, the distortion function for delta-delta features can be expressed as

$$
\Delta^{2} \mathbf{Y}=\mathbf{O} \bullet \Delta^{2} \mathbf{X}+\mathbf{E} \bullet \Delta^{2} \mathbf{N}+\mathbf{S} \bullet \mathbf{W} .
$$

It can then be derived that the mean vector and covariance matrix for delta-delta features can be compensated as follows:

$$
\begin{aligned}
\mu_{\Delta^{2} \mathbf{y}}= & \mu_{\mathbf{o}} \bullet \mu_{\Delta^{2} \mathbf{x}}+\mu_{\mathbf{e}} \bullet \mu_{\Delta^{2} \mathbf{n}}+\mu_{\mathbf{s}} \bullet \mu_{\mathbf{w}} \\
\Sigma_{\Delta^{2} \mathbf{y}}= & \Sigma_{\mathbf{0} \Delta^{2} \mathbf{x}}+\Sigma_{\mathbf{e} \bullet \Delta^{2} \mathbf{n}}+\Sigma_{\mathbf{s} \bullet \mathbf{w}} \\
& +\operatorname{Cov}\left(\mathbf{O} \bullet \Delta^{2} \mathbf{X}, \mathbf{E} \bullet \Delta^{2} \mathbf{N}\right)+\operatorname{Cov}\left(\mathbf{E} \bullet \Delta^{2} \mathbf{N}, \mathbf{O} \bullet \Delta^{2} \mathbf{X}\right) \\
& +\operatorname{Cov}\left(\mathbf{O} \bullet \Delta^{2} \mathbf{X}, \mathbf{S} \bullet \mathbf{W}\right)+\operatorname{Cov}\left(\mathbf{S} \bullet \mathbf{W}, \mathbf{O} \bullet \Delta^{2} \mathbf{X}\right) \\
& +\operatorname{Cov}\left(\mathbf{E} \bullet \Delta^{2} \mathbf{N}, \mathbf{S} \bullet \mathbf{W}\right)+\operatorname{Cov}\left(\mathbf{S} \bullet \mathbf{W}, \mathbf{E} \bullet \Delta^{2} \mathbf{N}\right),
\end{aligned}
$$

where

$$
\begin{aligned}
\Sigma_{\mathbf{o} \bullet \Delta^{2} \mathbf{x}} & =\Sigma_{\mathbf{o}} \bullet \Sigma_{\Delta^{2} \mathbf{x}}+\mu_{\mathbf{o}} \mu_{\mathbf{o}}^{T} \bullet \Sigma_{\Delta^{2} \mathbf{x}}+\Sigma_{\mathbf{o}} \bullet \mu_{\Delta^{2} \mathbf{x}} \mu_{\Delta^{2} \mathbf{x}}^{T},(19) \\
\Sigma_{\mathbf{e} \bullet \Delta^{2} \mathbf{n}} & =\Sigma_{\mathbf{e}} \bullet \Sigma_{\Delta^{2} \mathbf{n}}+\mu_{\mathbf{e}} \mu_{\mathbf{e}}^{T} \bullet \Sigma_{\Delta^{2} \mathbf{n}}+\Sigma_{\mathbf{e}} \bullet \mu_{\Delta^{2} \mathbf{n}} \mu_{\Delta^{2} \mathbf{n}}^{T},(20) \\
\Sigma_{\mathbf{s} \bullet \mathbf{w}} & =\Sigma_{\mathbf{s}} \bullet \Sigma_{\mathbf{w}}+\mu_{\mathbf{s}} \mu_{\mathbf{s}}^{T} \bullet \Sigma_{\mathbf{w}}+\Sigma_{\mathbf{s}} \bullet \mu_{\mathbf{w}} \mu_{\mathbf{w}}^{T},
\end{aligned}
$$

and

$$
\begin{aligned}
\operatorname{Cov}(\mathbf{E}, \mathbf{S}) & =-\operatorname{Cov}(\mathbf{O}, \mathbf{S}), \\
\operatorname{Cov}\left(\mathbf{O} \bullet \Delta^{2} \mathbf{X}, \mathbf{E} \bullet \Delta^{2} \mathbf{N}\right) & =\left(\operatorname{Cov}\left(\mathbf{E} \bullet \Delta^{2} \mathbf{N}, \mathbf{O} \bullet \Delta^{2} \mathbf{X}\right)\right)^{T} \\
& =-\Sigma_{\mathbf{o}} \bullet \mu_{\Delta^{2} \mathbf{x}} \mu_{\Delta^{2} \mathbf{N}}^{T} \\
\operatorname{Cov}\left(\mathbf{O} \bullet \Delta^{2} \mathbf{X}, \mathbf{S} \bullet \mathbf{W}\right) & =\left(\operatorname{Cov}\left(\mathbf{S} \bullet \mathbf{W}, \mathbf{O} \bullet \Delta^{2} \mathbf{X}\right)\right)^{T} \\
& =\operatorname{Cov}(\mathbf{O}, \mathbf{S}) \bullet \mu_{\Delta^{2} \mathbf{x}} \mu_{\mathbf{W}}^{T}, \\
\operatorname{Cov}\left(\mathbf{E} \bullet \Delta^{2} \mathbf{N}, \mathbf{S} \bullet \mathbf{W}\right) & =\left(\operatorname{Cov}\left(\mathbf{S} \bullet \mathbf{W}, \mathbf{E} \bullet \Delta^{2} \mathbf{N}\right)\right)^{T} \\
& =\operatorname{Cov}(\mathbf{E}, \mathbf{S}) \bullet \mu_{\Delta^{2} \mathbf{N}} \mu_{\mathbf{W}}^{T} .
\end{aligned}
$$

In the above equations, $\mu_{\mathbf{s}}, \Sigma_{\mathbf{s}}, \mu_{\mathbf{w}}, \Sigma_{\mathbf{w}}, \operatorname{Cov}(\mathbf{O}, \mathbf{S})$ are estimated by using the same UT method as in HMM compensation for delta features.

\section{EXPERIMENTS AND RESULTS}

\subsection{Experimental Setup}

In order to verify the effectiveness of the proposed UT approaches to HMM compensation for both static and dynamic features and compare them to the traditional PMC- and VTS-based approaches, two sets of experiments as summarized in Table 1 are performed for the task of speaker independent recognition of connected digit strings on Aurora2 database. A full description of the Aurora2 database and a test framework is given in [3]. The experimental setup here is the same as in [4] except that 1) the power rather than the magnitude of the Fourier transform is used in the binning process for calculating the outputs of mel-scaled filterbanks, 2) 20 instead of 3 Gaussian components are used for each digit CDHMM state. Our CDHMM-based ASR system is trained from the "clean" speech data in Aurora2 database and a 39-dimensional feature vector is used, which consists of 13 MFCCs (including $C_{0}$ ) plus their first and second order derivatives. The delta and delta-delta features are extracted using linear regression method as detailed in [9]. Both training and recognition were performed by using the HTK [9] and the standard scripts provided by ETSI [3]. Because we focus our study in this paper on HMM compensation for additive noise only, no other compensation is performed to cope with other possible distortions.

In the first set of experiments labeled as \#2 to \#6 in Table 1, both HMM means and variances for static features are compensated by using the log-normal-approximation-based PMC, the first-order-approximation-based VTS, and our UT-based approaches proposed in [4], and HMM means for dynamic features are compensated by using the heuristic approach described in [1]. The single Gaussian model of additive noise in each test sentence is estimated from noise frames at the beginning and end of the sentence in cepstral domain by using an ML approach. In the second set of experiments labeled as \#7 to \#13 in Table 1, both HMM means and variances for static features are compensated by using the augmented UT approach proposed in [4], and the approach in [1] or the simplified UT approach proposed in this paper are used to compensate for HMM means and/or variances for delta- and/or delta-delta features. Another difference is that the single Gaussian model of additive noise in each test sentence is estimated from noise frames at the beginning and end of the sentence in cepstral domain by using MAP estimation with a prior PDF estimated from "non-speech" frames in the corresponding testing "environment". This is to simulate a scenario where a relatively good noise estimation is achievable. Because we assume that the background noise is stationary, we set $\mu_{\Delta \mathbf{n}}$ and $\mu_{\Delta^{2} \mathbf{n}}$ as zero vectors.

\subsection{Experimental Results}

Table 2 summarizes a performance (word accuracy in \%) comparison of different HMM compensation methods, where the performance is averaged over SNRs between 0 and $20 \mathrm{~dB}$ on each of the three different test sets of Aurora2 database, namely Set A, Set B and Set C. It is observed that all of our proposed UT-based approaches to HMM compensation for static MFCC features achieve a better performance in all the test sets than that of both the PMCand VTS-based methods.

Table 3 summarizes a performance (word accuracy in \%) comparison of three HMM compensation methods for delta-features averaged over SNRs between 0 and $20 \mathrm{~dB}$ on test Set A of Aurora2 database. It is observed that 1 ) neither method \#8 nor \#9 
Table 2. Performance (word accuracy in \%) comparison of different HMM compensation methods averaged over SNRs between 0 and $20 \mathrm{~dB}$ on three different test sets of Aurora2 database (R.E.R. stands for the relative error rate reduction in $\% v s$. the baseline performance without HMM compensation).

\begin{tabular}{|c|c|c|c|c|c|}
\hline Methods & Set A & Set B & Set C & Overall & R.E.R. \\
\hline$\# 1$ & 63.03 & 57.06 & 74.06 & 62.85 & N/A \\
\hline$\# 2$ & 84.36 & 83.22 & 85.68 & 84.17 & 60.50 \\
\hline$\# 3$ & 83.66 & 81.88 & 84.73 & 83.16 & 57.99 \\
\hline$\# 4$ & 85.59 & 84.18 & 87.42 & 85.40 & 63.56 \\
\hline$\# 5$ & 85.32 & 83.92 & 87.02 & 85.10 & 62.82 \\
\hline$\# 6$ & 85.67 & 84.20 & 87.31 & 85.41 & 63.59 \\
\hline
\end{tabular}

Table 3. Performance (word accuracy in \%) comparison of different compensation methods for delta features averaged over SNRs between 0 and $20 \mathrm{~dB}$ on test Set A of Aurora 2 database.

\begin{tabular}{|c|c|c|c|c|c|}
\hline Methods & Subway & Babble & Car & Exhibition & Average \\
\hline$\# 7$ & 88.22 & 85.38 & 86.11 & 83.50 & 85.80 \\
\hline$\# 8$ & 87.04 & 83.32 & 86.54 & 82.34 & 84.81 \\
\hline$\# 9$ & 89.00 & 83.41 & 88.49 & 84.73 & 86.41 \\
\hline
\end{tabular}

works for the compensation of HMM variances for delta-features in nonstationary noisy environment (Babble); 2) in relatively more stationary noisy environments, namely Subway, Car, Exhibition, our proposed simplified UT approach (method \#9) works well for both HMM mean and variance compensation for delta-features, while the method in [1] only works well for HMM mean compensation for delta-features (method \#7), but not for HMM variance compensation for delta-features (method \#8). Similar observations are also made for noise environments in test Set B not shown here. Table 4 summarizes the detailed relative error rate reductions (in $\%$ ) of the method \#9 vs. method \#7 under different combinations of SNR and noise type of the test Set A on Aurora2 database.

Table 5 summarizes a performance (word accuracy in \%) comparison of four HMM compensation methods for both delta and delta-delta features averaged over SNRs between 0 and $20 \mathrm{~dB}$ on test Set A of Aurora2 database. It is observed that 1) neither method \#11 nor \#12 works for the compensation of HMM variances for delta-delta features; 2) method \#10 achieves the best performance in Subway and Babble environments, while method \#13 achieves the best performance in Car and Exhibition environments.

\section{CONCLUSION AND FUTURE WORKS}

Based on the previous results, we draw the following conclusion: 1) UT-based approach works well for HMM compensation corresponding to static MFCC features, and outperforms the log-normalapproximation-based PMC and first-order-approximation-based VTS approaches; 2) No good solution has been found yet for HMM compensation corresponding to dynamic features. Ongoing and future works include 1) to use UT-based approach for estimating a better noise model from the whole noisy testing utterance instead of just using the leading and ending frames. With a better noise model, it would be interesting to repeat the above experiments and see what will happen; 2) to extend UT methods for HMM compensation to cope with both convolutional and additive distortions; 3) to apply UT-based techniques for feature compensation; 4) to develop UT-based techniques for compensation of environmental variabilities in both training and recognition stages; 5) to develop UT-based techniques for dealing with nonstationary distortions; 6)
Table 4. Summary of relative error rate reductions (in \%) of method \#9 vs. method \#7 under different combinations of SNR and noise type on the test Set A of Aurora2 database.

\begin{tabular}{|c|c|c|c|c|c|}
\hline & Subway & Babble & Car & Exhibition & Average \\
\hline $20 \mathrm{~dB}$ & 11.63 & -19.01 & 24.59 & 0.00 & 4.30 \\
\hline $15 \mathrm{~dB}$ & 18.15 & -19.43 & 26.36 & 14.99 & 10.02 \\
\hline $10 \mathrm{~dB}$ & 10.91 & -28.83 & 15.26 & 8.83 & 1.54 \\
\hline $5 \mathrm{~dB}$ & 4.11 & -13.60 & 16.07 & 7.76 & 3.58 \\
\hline $0 \mathrm{~dB}$ & 5.74 & -10.21 & 17.04 & 6.72 & 4.82 \\
\hline Average & 6.62 & -13.47 & 17.12 & 7.48 & 4.26 \\
\hline
\end{tabular}

Table 5. Performance (word accuracy in \%) comparison of different compensation methods for delta-delta features averaged over SNRs between 0 and $20 \mathrm{~dB}$ on test Set A of Aurora2 database.

\begin{tabular}{|c|c|c|c|c|c|}
\hline Methods & Subway & Babble & Car & Exhibition & Average \\
\hline$\# 10$ & 90.32 & 83.27 & 88.94 & 86.42 & 87.24 \\
\hline$\# 11$ & 80.55 & 74.52 & 81.45 & 78.30 & 78.70 \\
\hline$\# 12$ & 87.80 & 81.58 & 88.29 & 85.55 & 85.80 \\
\hline$\# 13$ & 89.78 & 80.86 & 90.71 & 87.64 & 87.25 \\
\hline
\end{tabular}

to evaluate the above techniques on other tasks and databases. We will report those results elsewhere when they become available.

\section{REFERENCES}

[1] A. Acero, L. Deng, T. Kristjansson, and J. Zhang, "HMM adaptation using vector Taylor series for noisy speech recognition," in Proc. ICSLP, Beijing, 2000, pp. 869-872.

[2] M. J. F. Gales, Model-based Techniques For Noise Robust Speech Recognition, Ph.D. thesis, Cambridge University, UK, 1995.

[3] H. G. Hirsch and D. Pearce, "The Aurora experimental framework for the performance evaluation of speech recognition systems under noisy conditions," in Proc. ISCA ITRW ASR, Paris, France, Sep. 2000, pp.181-188.

[4] Y. Hu and Q. Huo, "An HMM compensation approach using unscented transformation for noisy speech recognition," in Proc ISCSLP-2006, Singapore, Dec. 2006.

[5] S. J. Julier and J. K. Uhlmann, "Unscented filtering and nonlinear estimation," Proceedings of the IEEE, Vol. 92, No. 3, pp.401-422, 2004.

[6] D.-Y. Kim, C.-K. Un, and N.-S. Kim, "Speech recognition in noisy environments using first-order vector Taylor series," Speech Cоттиnication, Vol. 24, pp.39-49, 1998

[7] P. J. Moreno, Speech Recognition in Noisy Environments, Ph.D. thesis, Carnegie Mellon University, 1996.

[8] P. J. Moreno, B. Raj, and R. M. Stern, "A vector Taylor series approach for environment-independent speech recognition," in Proc. ICASSP, Atlanta, 1996, pp.733-736.

[9] S. J. Young, et al., The HTK Book (revised for HTK Version 3.3), 2005. 